\title{
The origin of nitrogen: the implications of very metal poor stars
}

\author{
Cristina Chiappini ${ }^{1}$, Francesca Matteucci ${ }^{2}$ and Silvia K. Ballero ${ }^{2}$ \\ ${ }^{1}$ Osservatorio Astronomico di Trieste, OAT/INAF - Via G. B. Tiepolo 11, Trieste, Italy \\ email: chiappini@ts.astro.it \\ ${ }^{2}$ Dipartimento di Astronomia dell'Universita' di Trieste - Via G. B. Tiepolo 11, Trieste, Italy
}

\begin{abstract}
Our understanding on the nitrogen origin has recently greatly changed. New data on nitrogen abundances in very metal-poor stars $(-4<[\mathrm{Fe} / \mathrm{H}]<-3)$ show a quite surprising result: a high $\mathrm{N} / \mathrm{O}$ ratio suggestive of high levels of production of primary nitrogen in massive stars. Moreover, none of the stars measured so far has N/O ratios as low as the ones observed in Damped Lyman- $\alpha$ systems (DLAs) (currently the systems showing the lowest N/O ratios in the universe). We studied the implications of the above new data set for our understanding on the nitrogen enrichment in the Milky Way. We find that, to explain the new observations, we need to adopt stellar yields computed with stellar models in which rotation is taken into account and assume that stars born at $\mathrm{Z}<10^{-5}$ contribute a lot more $\mathrm{N}$ than the most recent calculations available in the literature for $Z=10^{-5}$. The implications of our findings for our understanding of the nature of Damped Lyman- $\alpha$ systems (DLAs) are also briefly discussed.
\end{abstract}

Keywords. Galaxy: abundances, Galaxy: halo, stars: rotation

\section{Results and Discussion}

The new data for nitrogen of Spite et al. (2005, this conference) have a profound impact on galactic evolution. Before this new data set, studies on the production of $\mathrm{N}$ in low metallicity environments were confined to the abundances measured in HII regions located in the outer disks of spiral galaxies, in metal poor dwarf galaxies or in DLAs. However, more stringent constraints on nitrogen nucleosynthesis come from the study of nitrogen abundances in stars in the Milky Way since they represent a true evolutionary sequence, where the stars with lower metallicity are the oldest ones. Moreover, the halo very metal poor stars play a fundamental role since below $[\mathrm{Fe} / \mathrm{H}]=-3$, only type II SNe have had time to enrich the interstellar medium from which these stars formed, thus offering a way to constrain the nitrogen production in massive stars at low metallicities. As shown by Chiappini et al. (2003a), the data available by then were in agreement with chemical evolution models adopting stellar yields where the nitrogen production in massive stars was only of secondary origin. As a consequence, these models predicted an increasing $\mathrm{N} / \mathrm{O}$ or $\mathrm{N} / \mathrm{Fe}$ abundance ratios as functions of metallicity. This behaviour persisted even when adopting the stellar yields of Meynet \& Maeder (2002, MM02) including rotation where the nitrogen production is increased and some primary $\mathrm{N}$ is produced in massive stars (solid line in Fig. 1 - Chiappini et al. 2003b).

The new data suggest an important primary nitrogen production in very metal poor stars. As shown by Chiappini et al. (2005), chemical evolution models computed with the stellar yields currently available in the literature cannot explain such high N/O ratios at low metallicities. However, we have shown that the new data can be explained by adopting the stellar yields obtained from stellar models which take into account rotation together with an extra production of $\mathrm{N}$ from massive stars born at metallicities $\mathrm{Z}$ below $10^{-5}$. In 


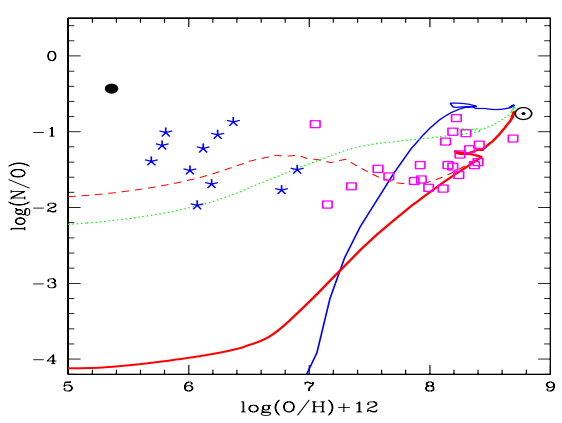

Figure 1. The data: Spite et al. (2005, asterisks) and Israelian et al. (2004, squares). The solid line represents a model computed with MM02 stellar yields. The dashed line shows the same model but with the $\mathrm{N}$ yields increased as suggested in Chiappini et al. (2005 - see text).

particular, we suggested an increase of nearly a factor of $200 \mathrm{in} \mathrm{N}$ for a star of $60 \mathrm{M}_{\odot}$ and around 40 for a star of $9 \mathrm{M}_{\odot}$ with respect to the yields given by MM02 for $\mathrm{Z}=10^{-5}$ and rotational velocity of $300 \mathrm{~km} / \mathrm{s}$. A model computed with the latter prescriptions can well fit the data and is shown in Fig.1 (dashed line). These prescriptions turned out to be in good agreement with the new calculations presented during this symposium by Hirshi for $\mathrm{Z}=10^{-8}$ and Ekström et al. for $\mathrm{Z}=0$ (these proceedings) and not yet published. Interestingly, we found values of the same order of magnitude by completely independent approaches.

Finally, if our results are confirmed, then it might be that only stars at very low metallicities contribute such large amounts of nitrogen. If this is the case, it is possible to explain the lowest $\mathrm{N} / \mathrm{O}$ ratios observed in some DLAs. DLAs have $[\mathrm{Fe} / \mathrm{H}]>-2.5$ whereas the halo stars of Spite et al. have $[\mathrm{Fe} / \mathrm{H}]<-3$. Therefore it is possible that in DLAs the high $\mathrm{N}$ production discussed above never took place. The lower limit on the DLAs metallicity might imply that these systems have been pre-enriched before the starting of the star formation. This is easier to envisage in the case of outer spiral disks as progenitors of DLAs because, as shown by Chiappini et al. (2001) for the Milky Way, the outer thin disk could have been pre-enriched by the halo/thick disk gas. Moreover, due to the gas density threshold, the star formation at large galactocentric distances should have been very inefficient, proceeding in bursts of short duration. Models computed with a bursting star formation are able to explain the bimodal distribution of DLAs in a N/O vs. $\mathrm{O} / \mathrm{H}$ diagram (see Prochaska, these proceedings) as being the result of different burst durations (see Chiappini et al. 2003b) rather than due to an age difference.

\section{Acknowledgements}

C. C and F. M. acknowledge partial financial support from the italian MIUR/Cofin 2003, 2003020839. C. C. acknowledges partial financial support through an IAU grant.

\section{References}

Chiappini, C., Matteucci, F. \& Ballero, S. K. 2005, A\&A in press (astro-ph/0503492)

Chiappini, C., Romano, D. \& Matteucci, F. 2003a, MNRAS 339, 63

Chiappini, C., Matteucci, F. \& Meynet, G. 2003b, A\&A 410, 257

Chiappini, C., Matteucci, F. \& Romano, D. 2001, ApJ 554, 1044

Israelian, G., Ecuvillon, A., Rebolo, R. et al. 2004, A\&A 421, 649

Meynet, G. \& Maeder, A. 2002, A\&A, 390, 561

Spite, M., Cayrel, R., Plez, B., Hill, V., Spite, F. et al. 2005, A\&A 430, 655 